\title{
BAND STRUCTURE AND REFRACTIVE INDEX OF GALLIUM NITRIDE UNDER PRESSURE
}

\author{
P. Perlin, I. Gorczyca, T. Suski, H. Teisseyre, I. Grzegory \\ High Pressure Research Center, Polish Academy of Sciences \\ Sokolowska 29/37, 01-142 Warszawa, Poland
}

AND N.E. Christensen

Institute of Physics, Aarhus University, Aarhus, Denmark

\begin{abstract}
The effect of hydrostatic pressure on direct gap and refractive index of $\mathrm{GaN}$ is investigated up to $5.5 \mathrm{GPa}$. Band structure of $\mathrm{GaN}$ is calculated by Linear Muffin-Tin Orbitals (LMTO) method for different values of pressure. Resulting pressure coefficient of the main gap and of the refractive index are in a good agreement with the experimental ones.
\end{abstract}

PACS numbers: 71.25.Tn, 78.20.Ci

\section{Introduction}

Many physical properties of semiconductors can be scaled with such material parameters as atomic volume and ionicity. Theory proposed by Van Vechten [1] was built upon this fact, and gave qualitatively good picture of properties of a great number of tetrahedrally coordinated semiconductors, including directly also pressure effects. From this point of view it seems interesting to investigate pressure behavior of GaN, which is the most ionic semiconductor in the III-V group ( 0.43 or $0.5[1,2]$ in the Phillips scale [3]) and has a very small atomic volume - about one half of that of GaAs. In the present work we measured the pressure dependence of absorption edge and refractive index (electronic dielectric constant).

The band structure of GaN is calculated from first principles the LMTO method within the LDA (Local Density Approximation). From the band structure for different values of pressure the pressure coefficient of the main gap is obtained and the pressure dependence of the refractive index is estimated. 


\section{Samples and experiment}

GaN single crystals have been grown with the use of a high pressure, high temperature method [4]. The samples we used were typically $100 \mu \mathrm{m} \times 20 \mu \mathrm{m}$ platelets. Experiment was performed in Merrill-Bassett-like diamond anvil cell with 4:1 methanol-ethanol mixture as a pressure transmitting medium under pressure up to $5.5 \mathrm{GPa}$, at room temperature. Both kind of experiments were performed in the transmission configuration.

\section{Results}

\subsection{Band gap}

GaN crystallizes in the wurtzite structure. The energy gap at ambient pressure varies from $3.41 \mathrm{eV}$ to $3.46 \mathrm{eV}$ depending on electron concentration. Pressure dependence of the absorption edge is shown in Fig. 1, showing typical sublinear dependence and can be well described by the equation $E_{0}(p)=-0.0018 p^{2}+0.047 p+$ 3.455 where $p$ is in $\mathrm{GPa}$ and $E_{0}$ in $\mathrm{eV}$. This result is in agreement with result pre-

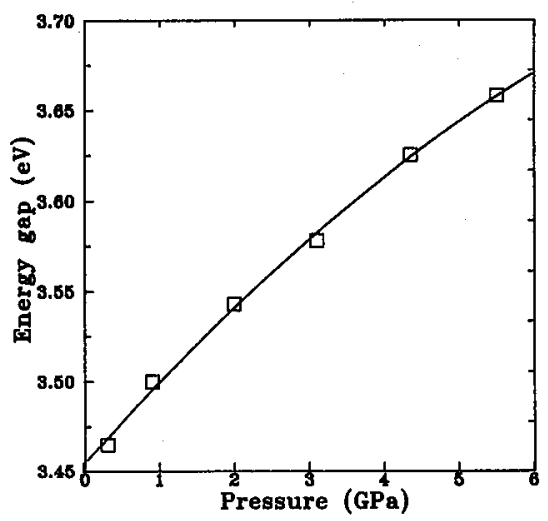

Fig. 1. Energy gap of GaN under pressure.

viously obtained by Camphausen [5] (linear coefficient equal to $0.042 \mathrm{eV} / \mathrm{GPa}$ in the pressure region up to $1 \mathrm{GPa}$ ) and is lower than that predicted in Van Vechten theory (0.053 eV/GPa [2]). Calculated band structure of GaN is shown in Fig. 2. General features of the band structure are the same as resulting from the pseudopotential calculations [6-8], but contrary to the latest results [8] the main energy gap is direct. Calculated pressure coefficient of the energy gap is equal $4.2 \mathrm{meV} / \mathrm{kbar}$ which is in a good agreement with the above experiment. Discrepancy with Van Vechten's [2] prediction can be easily explained by the fact that nearest-neighbor distance scaling is not very precise since its value is extremely small in GaN. 


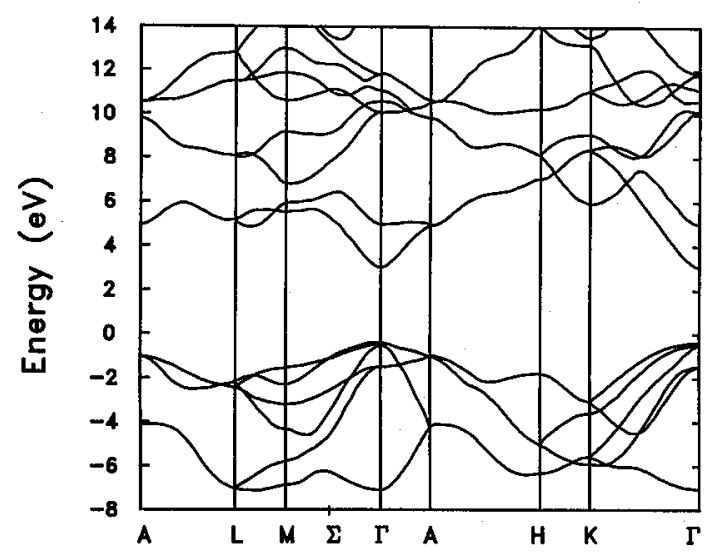

Fig. 2. Calculated band structure of $\mathrm{GaN}$ at ambient pressure.

\subsection{Refractive index}

If light passes through plane-parallel platelet one can observe oscillation spectrum in transmission where positions of maxima are given by equation $2 d n=$ $\alpha \lambda_{\alpha}$; here $d$ is the thickness of the sample, $\alpha$ is number of given maximum and $\lambda_{\alpha}$ is a wavelength corresponding to this maximum. Under pressure we can follow any interference maximum and thus determine the behavior of $n(p)$. Compression of the sample is included in calculations with the use of equation of state [9]. Figure 3 shows the pressure dependence of refractive index. We can see that $n$

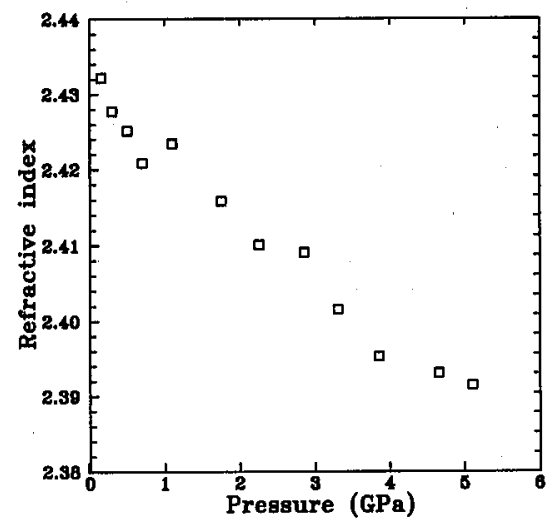

Fig. 3. Pressure dependence of refractive index of $\mathrm{GaN}$ for $\lambda=900 \mathrm{~nm}$.

decreases like in the majority of other compounds. The linear pressure coefficient $(1 / n)(\mathrm{d} n / \mathrm{d} p)$ is equal $-0.2 \times 10^{-6} \mathrm{bar}^{-1}$. This result differs importantly from the value $-0.05 \times 10^{-6}$ predicted by Camphausen [2]. Taking the results of performed 
band structure calculations and using the following formula resulting from the Penn model [10]:

$$
\frac{1}{n} \frac{\partial n}{\partial p}=\frac{1}{2}\left(1-\varepsilon^{-1}\right)\left(K-2 E_{0}^{-1} \frac{\partial E_{0}}{\partial p}\right)
$$

(where $K$ is the isothermal compressibility and $E_{0}$ is average Penn gap) we can estimate the theoretical value of $(1 / n)(\mathrm{d} n / \mathrm{d} p)$. Following considerations given by Tsay et al. [11] we assume that the average Penn gap occurs at the $\Sigma$ point of $\mathrm{BZ}$ (see Fig. 3). We have got the energy gap at this point equal to $7.42 \mathrm{eV}$ and its pressure coefficient equal to $4.0 \mathrm{meV} / \mathrm{kbar}$. With the above values we obtain $(1 / n)(\mathrm{d} n / \mathrm{d} p)=-0.27 \times 10^{-6} \mathrm{bar}^{-1}$, which is in very good agreement with the value obtained experimentally. Previous theoretical calculations predicted negative value of $\mathrm{d} n / \mathrm{d} p$ for III-V compounds, positive for II-VI and nearly 0 for $\mathrm{GaN}$ which is intermediate in some sense. Cur result does not confirm this trend. It is worth noting that for tetrahedrally coordinated compounds the experimental values of $\mathrm{d} n / \mathrm{d} p$ are always negative.

\section{References}

[1] J.A. Van Vechten, Phys. Rev. 182, 891 (1969).

[2] D.L. Camphausen, G.A. Neville Conell, W. Paul, Phys. Rev. Lett. 26, 184 (1971).

[3] J.C. Phillips, Phys. Rev. Lett. 20, 550 (1968).

[4] S. Porowski, I. Grzegory, J. Jun, in High Pressure Chemical Synthesis, Elsevier, Amsterdam 1989.

[5] D.L. Camphausen, G.A.N. Conell, J. Appl. Phys. 42, 4438 (1971).

[6] D. Jones, A.H. Lettigton, Solid State Commun. 23, 815 (1972).

[7] S. Bloom, G. Harbeke, E. Meier, I.B. Ortenburg, Phys. Status Solidi B 66, 161 (1974).

[8] S.H. Grinaiev, V.J. Malakhov, V.A. Tschaldishev, Izv. Vyssh. Uchebn. Zaved. Fiz. (USSR) 29, 69 (1986).

[9] F. Perlin, C. Jauberthie-Carillon, J.P. Itie, A. San Miguel, I. Grzegory, A. Polian, in Proc. XXVIII EHPRG Conf., Bordeaux 1990.

[10] D.R. Penn, Phys. Rev. 128, 2093 (1962).

[11] Y.F. Tsay, S.S. Mitra, B. Bendow, Phys. Rev. B 10, 1476 (1974). 